\title{
BH3 Only Proteins as Major Player in Treatment of Skin Melanoma
}

\author{
Puneet Gupta and Jyoti Vakhlu* \\ School of Biotechnology, University of Jammu, India
}

Received: 㘹: September 28, 2018; Published: 琒 October 04, 2018

*Corresponding author: Jyoti Vakhlu, School of Biotechnology, University of Jammu, Jammu, (J\&K), India

\section{Mini Review}

Skin epidermis is made up of stratified epithelium. Stratification plays an important role during epidermal development. Asymmetric cell division occurs in the single layer of basal cells followed by stratification leads to production of committed suprabasal during epidermal development. Suprabasal cells thus formed are immature and have to undergo cell divisions many times to form fully stratified epithelia. There is a balance between cell growth and cell death due to homeostasis, which plays a vital role in development and maintenance of multicellular organisms. Homeostasis is controlled by various molecular mechanisms including apoptosis. UVB irradiation generally causes DNA damage leading to activation of the intrinsic cell death pathway which includes mitochondrial damage and cytochrome c release in a receptor-independent way. Cellular proteins such as p53 and Bax play major role in the intrinsic pathway whereas mitochondria promote apoptosis by controlling apoptosome-mediated activation of caspase-9.

Apoptosis is generally defined as programmed cell death responsible for elimination of damaged and redundant cells by activation of various type of caspases such as aspartate-specific and cysteine proteases. Caspases cleave important proteins, leading to fragmentation of cells into vesicles that can undergo phagocytosis. In mammalian cells, apoptosis can be triggered by two pathways i.e. extrinsic pathway and intrinsic pathway. Extrinsic pathway involves engagement of cell surface receptors of tumor necrosis factor receptor family. On the other hand, intrinsic pathway which is also known as mitochondrial pathway is generally triggered by various cellular stresses. Interactions between BCL2 and its homologues sharing BCL2 homology (BH) domains controls intrinsic pathway. BCL2 along with its homologues BCL-XL, MCL1, BCL-W, and A1 inhibits apoptosis. Other related proteins such as BAX, BOK that are similar in sequence and structure promotes apoptosis. Distant related proteins such as BID BIM, PUMA, NOXA, BMF, BAD, BIK and HRK which have only the BH3 domain in common with BCL2 promotes apoptosis. Stress results in activation of BCL2 related proteins having $\mathrm{BH} 3$ domain triggers apoptosis by neutralizing the pro-survival proteins.
Conformational change and homo-oligomerization in activated BAX and BAD leads to permeabilization of outer mitochondrial membrane followed by subsequent release of cytochrome $c$ into the cytoplasm results in the formation of the apoptozole complex, which in turn activates initiator caspase-9. BH3 domain is an amphipathic $\alpha$-helix consisting of 26 -residues that binds tightly to hydrophobic groove on the surface of BCL2 pro-survival members. Individual BH3-only proteins show different affinities for different pro-survival proteins. Till date molecular mechanism by which BH3 only proteins induce cell death except BNIP3 is not known. Recent studies show that hypoxia can induce BH3 only proteins in certain tumor cells and induction of BH3 only proteins is directly proportional with cell death. Efficient apoptosis requires neutralization of all pro-survival BCL2 family members expressed within a given cell. There is still open debate on how the BH3-only proteins promotes activation of BAX and BAK related proteins. BH3-only proteins are regulated by various molecular mechanisms that can be transcriptional as well as post-translational.

Apart from BIM, Loss of individual BH3-only proteins shows relatively normal phenotype in the absence of stress conditions confirms functional overlap. Individual cytotoxic conditions induce unique, overlapping, expression patterns that can vary with particular cell type. Inhibition of apoptosis is a fundamental step in tumor development whereas induction of apoptosis might be responsible for tumor suppression. Genes encoding BH3-only proteins have been found mutated in various human cancers. Experiment using knocked out mice have proved that BH3-only proteins have the capacity to serve as tumor suppressors. BH3-only proteins play a vital role in the elimination of tumor cells by various anti-cancer agents. Now a days, BH3-only proteins mimetics are being employed in treatment of various human cancers. BH3 mimetics such as ABT-737 and its orally active derivative ABT263 have the tendency to kill tumor cells through a BAX- and BAK-dependent mechanism. BH3-only proteins are prerequisite for triggering apoptosis using mitochondrial pathway. Prediction of proapoptotic BH3-only proteins which are responsible for 
elimination of specific tumor cells can provide valuable insights into difference therapeutic efficacy by conventional and targeted anti-cancer agents. In the present era, knowledge of BH3 mimetics can be exploited in the treatment of skin melanoma with least damage to normal tissues.

\section{ISSN: 2574-1241}

DOI: $10.26717 / B J S T R .2018 .09 .001826$

Jyoti Vakhlu. Biomed J Sci \& Tech Res

(c) (P) This work is licensed under Creative

Submission Link: https://biomedres.us/submit-manuscript.php

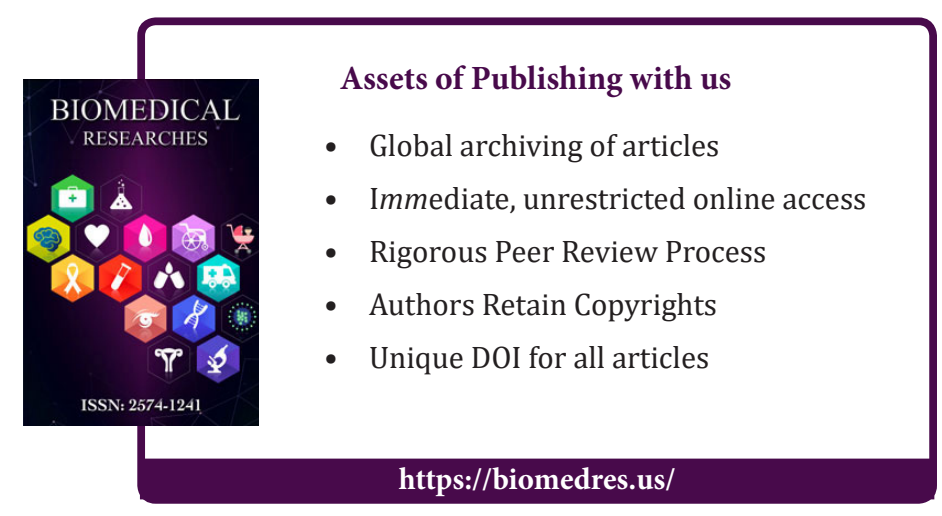

